\title{
Antibiotic prophylaxis in ragged placental membranes: a prospective, multicentre, randomized trial
}

\author{
Hian Yan Voon ${ }^{1,24^{*}}$ D, Jun Yan Pow ${ }^{1}$, Lee Na Tan ${ }^{3}$, Haris Njoo Suharjono ${ }^{1,4}$ and Wan Sim Teo ${ }^{1}$
}

\begin{abstract}
Background: Ragged placental membranes is a distinct entity from retained placenta and not uncommonly reported in midwifery texts. Although the incidence of postpartum endometritis is merely $1-5 \%$ after vaginal births, it remains the most common source of puerperal sepsis, contributing up to $15 \%$ of maternal mortality in low income countries. Geographically-remote centres in Malaysia prophylactically administer antibiotics for women with ragged placental membranes after vaginal birth, extrapolating evidence from retained placenta. We sought to clarify the rationale in continuing such practices.

Methods: This was an open-label, prospective, multicentre, randomized trial. Three hospitals where the current protocol was to administer prophylactic amoxycillin-clavulanic acid served as the sites of recruitment. Women who delivered vaginally beyond $24^{+0}$ weeks of gestation with ragged membranes were invited to participate in the trial and randomized into prophylaxis or expectant management with medical advice by blocks of 10, at a 1:1 ratio. A medication adherence diary was provided and patients followed up at 2 weeks and 6 weeks postpartum.
\end{abstract}

Results: A total of 6569 women gave birth vaginally in three centres during the trial period, of which 10.9\% had ragged membranes. The incidence of endometritis was not significantly raised in women with or without prophylaxis $(0.90 \%$ vs $0.29 \% ; p=0.60)$. All cases of endometritis presented within the first 2 weeks and preventive use of antibiotics did not ameliorate the severity of endometritis since rates of ICU admission, surgical evacuation and transfusion were comparable.

Conclusion: Preventive use of antibiotics after vaginal delivery in women with ragged placental membranes did not result in a reduction of endometritis. Educating women on the signs and symptoms of endometritis would suffice. Based on the reported incidence of ragged membranes, a change in practice would result in 1500 less prescriptions of antibiotics per annum in these three centres.

Trial registration: NCT 03459599 (Retrospectively registered on 9 March 2018).

Keywords: Antibiotic, Endometritis, Placental membranes, Prophylaxis, Ragged membranes

\section{Background}

Puerperal sepsis is one of the leading direct causes of maternal mortality worldwide, as the process of childbirth inherently increases a woman's exposure to infections arising from the genital tract. The 2014 MBRRACE-UK (Mothers and Babies: Reducing Risk

\footnotetext{
* Correspondence: vhaxyn@gmail.com

${ }^{1}$ Department of Obstetrics and Gynaecology, Sarawak General Hospital, Hospital Road, 93586 Kuching, Sarawak, Malaysia

${ }^{2}$ Department of Obstetrics and Gynaecology, Sri Aman Hospital, Hospital Road, 95000 Sri Aman, Sarawak, Malaysia

Full list of author information is available at the end of the article
}

through Audits and Confidential Enquiries across the UK) report highlighted an unenviable fact that $25 \%$ of women who died had succumbed to sepsis [1]. While postpartum haemorrhage, obstetric embolism and hypertensive disorders remain the principal causes, an unsettling trend is also observed locally, with maternal deaths from puerperal sepsis doubling from 1.6 to $3.1 \%$ in the latest Malaysian Confidential Enquiries into Maternal Deaths [2].

Although the incidence of postpartum endometritis is merely $1-5 \%$ after vaginal births, it remains the most

(c) The Author(s). 2019 Open Access This article is distributed under the terms of the Creative Commons Attribution 4.0 International License (http://creativecommons.org/licenses/by/4.0/), which permits unrestricted use, distribution, and 
common source of puerperal sepsis $[3,4]$. It is estimated that postpartum endometritis contributes $15 \%$ of maternal mortality in low income countries. Long term complications from postpartum endometritis such as chronic pelvic inflammatory disease, ectopic pregnancy and subfertility not only lead to repeated health care visits but are substantial morbidities in survivors [5]. Among the reported risk factors for postpartum endometritis, prolonged rupture of membranes, use of internal fetal monitoring, operative vaginal delivery and caesarean section can be found. However, other than caesarean section, these risk factors did not appear to be significant predictors of subsequent infection [6].

Ragged placental membranes is a distinct condition from retained placenta or placental cotyledon and is often cited in midwifery texts. Its clinical significance lies in the potential for uterine subinvolution, haemorrhage and infection $[7,8]$. The presence of ragged membranes is routinely documented in delivery notes and codable by the International Classification of Diseases, 10th Revision (ICD-10), [9]. The literature reflects a distinct paucity of data specifically relating to ragged or retained membranes, resulting in the authors extrapolating information from prophylactic antibiotic use in proxy conditions such as retained placenta, operative vaginal delivery and even routine postnatal prophylaxis as described below.

The World Health Organization (WHO) recommends the use of antibiotics prior to manual removal of placenta but acknowledges the lack of randomized controlled trials to support it, as evidence is largely extrapolated from caesarean sections [10, 11]. Indeed the lack of data is surprising, given that it is a common obstetric complication. Chongsomchai and colleagues have not been able to identify any studies in their systematic review, back in 2006 and as recent as 2014 [12]. A recent meta-analysis of retrospective cohort studies was performed in an attempt to clarify this, showing a non-significant reduction of endometritis with prophylaxis (OR 0.84), although the definition of endometritis was not explicit and the results did not achieve statistical significance [13].

Interestingly, the use of antibiotic prophylaxis for women who deliver vaginally has also been examined in women after instrumental delivery, routinely after any vaginal delivery and even antenatally in women with risk factors for sexually transmitted disease [14-16]. In all the scenarios above, reductions in the incidence of endometritis were found. A Cochrane review identified one trial involving 393 women who required operative vaginal delivery, in which 7 developed endometritis without antibiotic prophylaxis while none had such complications in the treatment group. The trial was judged to be of low risk for bias but in the absence of additional evidence, the reviewers abstained from recommending prophylaxis [14].

A large French randomized trial recruited low risk women after vaginal delivery and administered a single dose of broad spectrum antibiotics before discharge. The authors found a four-fold reduction in endometritis with prophylaxis and were still able to demonstrate a $70 \%$ cost saving in such women, compared to subsequent treatment. The role of the pharmaceutical company was unclear and there was no disclosure by the authors [15].

Therefore, administering antibiotics to women adjudged to be at risk of endometritis, as evidenced by the presence of ragged membranes invites controversy. The hospitals invited to participate in this trial currently implement the protocol of giving amoxycillin-clavulanic acid as prophylaxis if ragged or retained membrane is present after vaginal birth. These hospitals serve a large patient catchment area in the Malaysian state of Sarawak and face considerably more logistical challenges than many hospitals in Peninsular Malaysia. While some of the hospitals serve as referral centres and are located in larger towns, the patients eventually return to more geographically-remote areas postnatally. This remains the principal reason behind the protocol of providing prophylaxis for many years, despite the lack of concrete evidence. An unpublished survey by the lead author reveals that similar practice does not apply in many hospitals in Peninsular Malaysia.

However, with significant improvements in rural health care facilities and telecommunications connectivity over the past decade, we wish to re-examine the role of continuation of such a protocol. Furthermore, finite financial resources and patient concerns about medicalization of obstetric care provides an additional impetus for this paper. Therefore the risk-benefit ratio of undertaking this trial is favourable.

\section{Methods}

This was an open-label, prospective, multicentre, randomized trial. Three hospitals in Sarawak where the current local protocol was to administer prophylactic amoxycillin-clavulanic acid served as the sites of recruitment. Eligible women were randomized to receive either prophylaxis, as part of the existing protocol (control) or no prophylaxis (intervention). Both groups of women were given medical advice regarding symptoms of endometritis as detailed below. No modifications to the dose, timing or mode of administration of the intended medication were made to the prophylaxis group.

Women who delivered vaginally beyond $24^{+0}$ weeks of gestation and were found to have ragged placental membranes were invited to participate in the trial. This was defined as placental membranes perceived to be incompletely removed by the accoucher during the third stage 
of labour, as opposed to delivery of membranes en bloc with the main placental mass. The appearance may involve irregular, scalloping edges of the membranes when inspected and is typically but not invariably, associated with pieces of membrane requiring evacuation from the cervical os (Figs. 1 and 2). When the completeness of placental membranes was in doubt, a second, more experienced accoucher would be consulted.

In all women where ragged placental membranes were diagnosed, immediate bedside digital evacuation from the dilated cervical os was attempted. Regardless of the amount of membranes removed, the diagnosis holds. However, if suspicion arose that a whole layer of chorionic or amniotic membrane was retained, formal assessment by the labour ward registrar was triggered.

Exclusion criteria were as follows:

a) Fever, within 5 days preceding delivery (axillary temperature $>37.5^{\circ} \mathrm{C}$ on 2 or more occasions at least $1 \mathrm{~h}$ apart or temperature $>38^{\circ} \mathrm{C}$ on one occasion). This also includes intrapartum fever

b) Required oral or intravenous antibiotics for any other obstetric-related (ex. third or fourth degree tears, preterm prelabour rupture of membranes) or non- obstetric related (ex. pneumonia, acute pyelonephritis) reasons

c) Retained placenta

d) Prolonged rupture of membrane ( $>18 \mathrm{~h}$ )

e) Retroviral disease, on long term oral or parenteral steroid or receiving other forms of immunosuppressants, including chemotherapy within the last one year

f) Vaginal delivery for an intrauterine death

g) Penicillin allergy

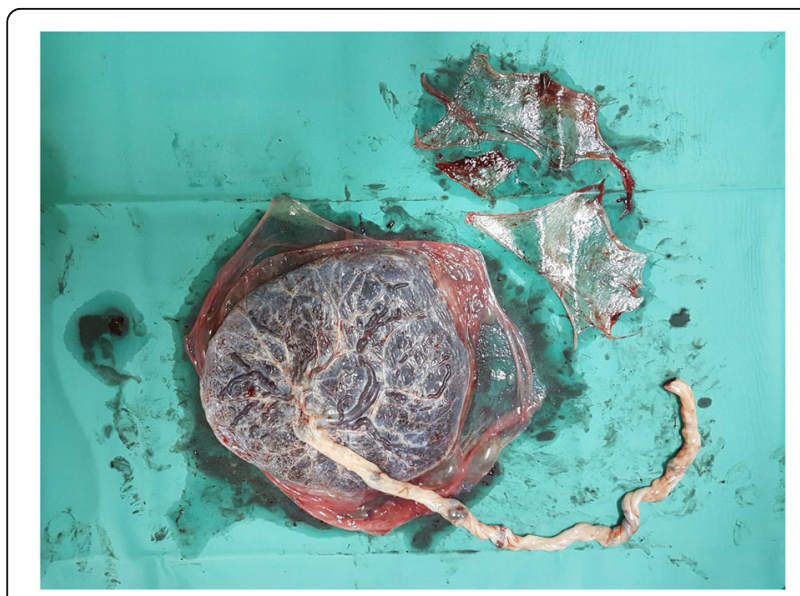

Fig. 1 Examination of the placental surface of placenta. Ragged membranes were evacuated separately and shown on the top right corner

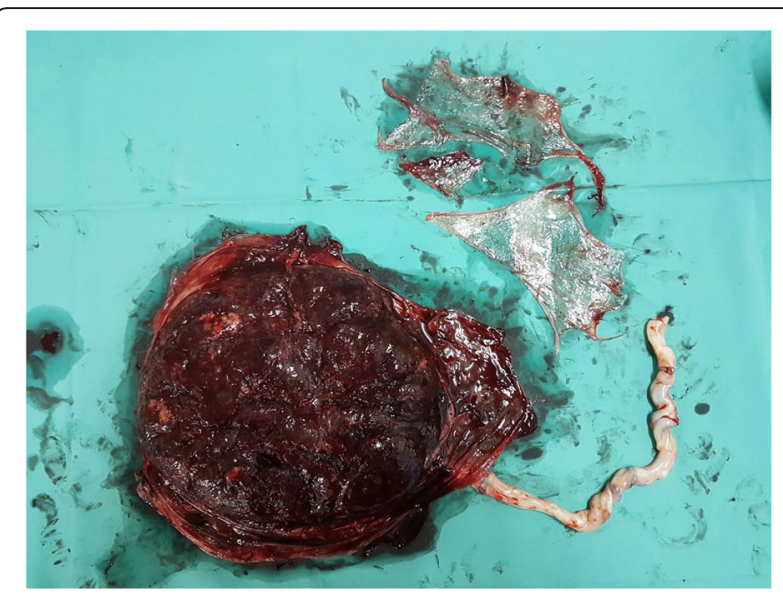

Fig. 2 Examination of the maternal surface of placenta. Ragged membranes were evacuated separately and shown on the top right corner

Recruitment took place in the labour suite of the respective hospitals, approximately an hour after delivery and perineal repair. The mothers were routinely counseled regarding red-flag symptoms suggestive of endometritis such as lower abdominal pain, increasing lochia loss and fever, as was the current practice. A patient information leaflet in either English or Malay was provided before they were transferred out of the labour suite. A second point of contact was made by investigators within 6-8 $\mathrm{h}$ to confirm their interest in participation. Written, informed consent were taken from women who agreed to participate before randomly assigning them on a ratio of 1:1 (prophylaxis arm or no prophylaxis arm) by block randomization (block size of 10), stratified by site of recruitment, using a web-based randomization program.

Women allocated to the prophylaxis arm received a week's course of amoxycillin-clavulanic acid tablets 625 mg three times a day, with the first dose administered within $6-8 \mathrm{~h}$ after delivery. A patient diary was provided to enhance their adherence to medication. This is an open label trial and did not involve the use of placebo. The author performing the analysis (VHY) was not involved in recruitment and remained blinded to the assigned arms. Women who declined participation would be excluded from the trial and given prophylaxis as per local protocol and advised on the red-flag symptoms of endometritis.

Upon discharge, follow-up telephone calls were conducted by the authors at 2 weeks and 6 weeks postdelivery. Medication adherence, discontinuation and side effects would be clarified. Symptoms of endometritis were enquired and if present, women were seen within $24 \mathrm{~h}$ in the Obstetric Daycare Unit of the respective hospitals. The follow-up telephone call would also allow the detection of any admission for endometritis in these women, should they be admitted to hospitals other than the ones involved in recruitment. 
Postpartum endometritis was defined as any intrauterine infection occurring after the third stage of labour until 6 weeks post delivery. At least 2 of the following symptoms or signs would have to be present at the time of diagnosis [5, 17]:

a) Fever (axillary temperature $>37.5^{\circ} \mathrm{C}$ on 2 or more occasions at least $1 \mathrm{~h}$ apart or temperature $>38^{\circ} \mathrm{C}$ on one occasion), occurring in the absence of apparent source of infection or alternative foci of infection.

b) Increasing lochia loss or offensive lochia.

c) Lower abdominal pain or suprapubic tenderness on palpation.

Alternatively, in the event that only one of the symptoms above was present, the diagnosis of endometritis could also be established by:

i. Positive genital swab culture in the presence of (a).

ii. Elevated total white cell count $>11.0 \times 10^{9} \mathrm{cells} / \mathrm{L}$ in the presence of (b) or (c).

Women who developed endometritis were treated as per the local hospital's protocol, at the discretion of the attending healthcare provider. This included outpatient treatment, admission for intravenous antibiotics or surgical evacuation. No additional serum monitoring for transaminitis was warranted for amoxycillin-clavulanic acid as this risk is low and there are no recommendations from the manufacturer. There were no changes to the methods after trial commencement. All information collected were transcribed to a pre-piloted proforma by the respective site coordinators. A single hard copy was kept in a designated locked compartment within the recruitment site, accessible only to the site coordinator and compiled into an electronic format at the end of the period of follow up. The electronic copy was devoid of patient identifiers. Once the information in the electronic copy was deemed to be complete and cross checked by coordinators from another site, the hard copy was destroyed as agreed upon during the ethical approval process.

We designed this trial to establish a 5-fold reduction in the incidence of endometritis from $5 \%$ in the interventional group (no prophylaxis) to $1 \%$ in the control group (prophylaxis continued as per protocol). The baseline rate of endometritis and reduction of the event with prophylaxis were estimated from existing studies $[4,15,18]$. The power of the trial (1-beta) was set at 0.85 with a significance level (alpha) of 0.05 . The sample size was calculated using PS software version 3.0.12 [19].

Three hundred twenty-five women on each arm would be required to reject the null hypothesis that antibiotic prophylaxis resulted in a five-fold reduction of postpartum endometritis after vaginal delivery in women who had ragged placental membranes.

Analysis was performed using SPSS 21.0 $0^{\text {tm }}$ (IBM). Descriptive analysis would be performed on patient demographics. Incidences of the primary outcomes (postpartum endometritis), secondary outcomes (ICU admission, surgical evacuation of products of conception and blood transfusion) were calculated. Categorical data were analysed using Chi square test and continuous data with independent T-test. Continuous variables which were not normally distributed, such as the duration of second stage were analysed using Mann-U Whitney test. Analysis was by intention-to-treat.

Approval from the Medical Research and Ethics Committee (MREC), Ministry of Health Malaysia was obtained prior to embarking on the trial (NMRR ID 161016-31,034). The trial was retrospectively registered with ClinicalTrials.gov (NCT 03459599).>

\section{Results}

A total of 6569 women delivered vaginally across three centres during the trial period, from March to September 2017 , of which $10.9 \%$ had ragged membranes. Recruitment was stopped when the required sample size was achieved. Figure 3 shows the CONSORT flow diagram from recruitment till analysis. A total of 670 patients were available for analysis, whereby 332 received prophylaxis and 338 did not. 11 women were excluded in the prophylaxis arm due to error in allocation, withdrawal of consent or loss of follow up. 17 women from the no prophylaxis arm were excluded for similar reasons but also included 6 women who required antibiotics due to other medical or obstetric indications. Sarawak General Hospital, Bintulu Hospital and Sri Aman Hospital contributed 577 (86.1\%), 61 (9.1\%) and $32(4.8 \%)$ patients respectively, proportional to the delivery delivery rates of each centre.

There were no differences between both groups in terms of age, parity, body mass index at booking, history of smoking, sexually-transmitted disease or previous preterm labour prior to 34 weeks. Mean gestational age at delivery, instrumental delivery, median second stage duration and the number of vaginal examinations were comparable (Table 1). Five women required internal fetal scalp electrode, all of whom were from the group that did not receive prophylaxis, while there were none in the prophylactic group.

The incidence of endometritis was not significantly raised in women with or without prophylaxis $(0.90 \%$ vs $0.29 \%, p=0.60$ ). All women diagnosed with endometritis based on the predefined criteria, presented within the first two weeks after delivery. Women in the prophylactic group presented between days 8-13 while the sole patient without prophylaxis presented much earlier, on day 2 . 


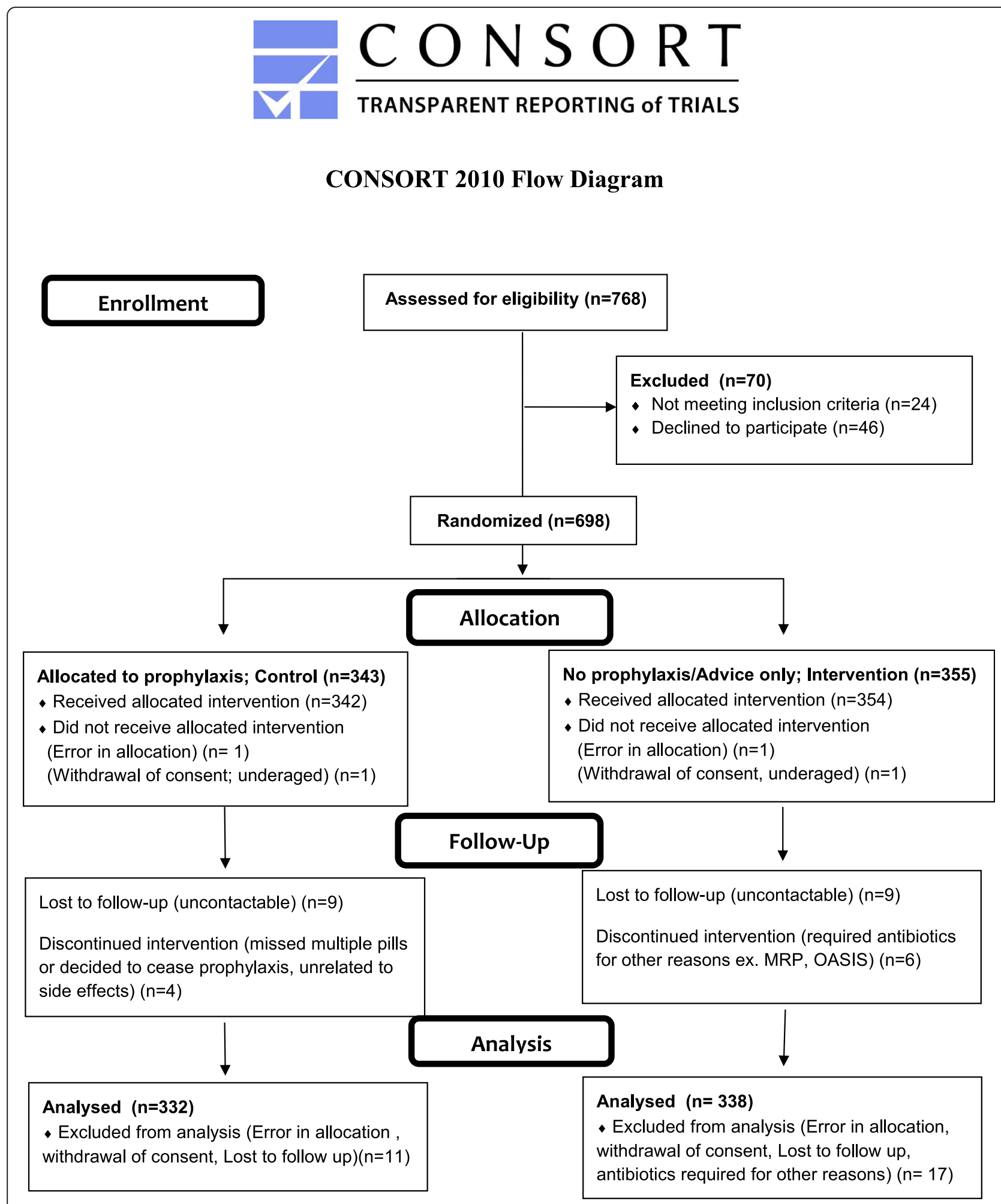

Fig. 3 Enrollment, randomization and follow up

Based on the diary kept by women who received prophylaxis, two of them were compliant while another missed six doses of medication. The latter felt that prophylaxis was unnecessary since she had been feeling well several days post delivery. No serious adverse effects were reported by patients receiving prophylaxis. 
Table 1 Baseline and intrapartum characteristics of trial participants

\begin{tabular}{|c|c|c|c|}
\hline Characteristics & Prophylaxis $n=332$ & No prophylaxis $n=338$ & $P$-value \\
\hline \multicolumn{4}{|l|}{ Baseline } \\
\hline Age (years) & $28.04(5.67)$ & $28.00(6.02)$ & 0.94 \\
\hline Parity (number) & $2.42(1.30)$ & $2.40(1.48)$ & 0.83 \\
\hline BMI $\left(\mathrm{kg} / \mathrm{m}^{2}\right)$ & $25.15(5.22)$ & $24.51(5.18)$ & 0.11 \\
\hline Smoking & $7(2.1 \%)$ & $4(1.2 \%)$ & 0.38 \\
\hline Previous preterm < 34 weeks & $10(3.0 \%)$ & $8(2.4 \%)$ & 0.60 \\
\hline Sexually transmitted disease & $0(0.0 \%)$ & $0(0.0 \%)$ & - \\
\hline \multicolumn{4}{|l|}{ Intrapartum } \\
\hline Gestation at delivery (weeks) & $38.63(1.68)$ & $38.61(1.67)$ & 0.84 \\
\hline Internal fetal monitoring & $0(0.0 \%)$ & $5(1.48 \%)$ & $0.06^{* *}$ \\
\hline Instrumental delivery & $16(4.80 \%)$ & 19 (5.20\%) & 0.64 \\
\hline Second stage duration $(\min )^{*}$ & 7 & 8 & 0.07 \\
\hline Number of vaginal examinations & $4(2)$ & $4(2)$ & 0.34 \\
\hline
\end{tabular}

Values represent mean (standard deviation) or as number (percentages)

* Represent median value

**Fisher exact test used

Intensive care unit admission, the need for blood transfusion and surgical evacuation were similarly low in both groups (Table 2). The average length of stay was three days.

\section{Discussion}

This trial was conducted as part of the state's on-going effort in keeping abreast with contemporaneous, evidencebased medicine, taking into account the improved accessibility of healthcare services. Such measures included elimination of unnecessary practices, videlicet antibiotic prophylaxis in ragged placental membranes.

The incidence of endometritis in our trial was similarly low regardless whether prophylaxis was given or not and the presentation of such cases was usually within the first fortnight post delivery. Although women who received prophylaxis appeared to present later, we were unable to conclude whether prophylaxis did in fact delay the presentation of endometritis due to the small number of women developing complications. The severity of endometritis did not appear to be ameliorated by the use of prophylaxis as there were no statistically significant differences in ICU admission, blood transfusion or need for surgical evacuation.
The findings in this trial was in contrast to the potential reduction in endometritis in other scenarios post-vaginal delivery, such as retained placenta and instrumental delivery described earlier [13, 14]. Interestingly, the role of antenatal antibiotic prophylaxis to prevent postpartum endometritis has also been studied in several randomized controlled trials. In fact, a systematic review and meta-analysis showed a $50 \%$ reduction in endometritis with prophylaxis, given between 26 and 32 weeks, although two thirds of the population included were deemed to be at high risk for sexually transmitted diseases [16]. However, it must be emphasized that such interventions were performed before the publication of the Oracle Children Study II in 2008. This landmark trial assessed children at the age of seven, showing an increased risk of cerebral palsy when their mothers were prescribed antibiotics for preterm labour and intact membranes [20]. Suffice to say, the attitude of obstetricians towards prescription of antibiotics antenatally has shifted since its publication. Furthermore, the incidence of sexually transmitted disease based on selfreported history or a positive serological or genital swab was low in our cohort.

Table 2 Outcomes of patients with ragged membranes

\begin{tabular}{llll}
\hline Complication & Prophylaxis $n=332$ & No prophylaxis $n=338$ & $P$-value \\
\hline Endometritis & $3(0.90)$ & $1(0.29)$ & 0.60 \\
ICU admission & 0 & 0 & $1(0.29)$ \\
Blood transfusion & $1(0.30)$ & 0 & 0.32 \\
Surgical evacuation & $1(0.30)$ & 0.50 \\
\hline
\end{tabular}

Values represent number (percentages); ICU Intensive care unit 
One contentious aspect of this trial was our consensus to use the term "prophylaxis" rather than "treatment", when in fact a full course of antibiotics was prescribed. The authors felt that treatment should be reserved for a recognized pathology rather than conditions of equipoise such as this. The reviewers aptly suggested we consider the term "preventive" use of antibiotics.

With approximately 13,500 vaginal deliveries per annum across these centres, it is estimated that a change in practice would see 1500 less women requiring superfluous treatment. This supports antimicrobial stewardship initiatives in preventing the emergence of antibiotic-resistant infections. Another area of concern with a more liberal policy on antibiotics is the alteration of neonatal gut microbiome with peripartum administration of antibiotics in breastfeeding women [21].

The strength of this trial included the prospective randomized design and a system for recall which allowed patients to be traced even if they were admitted to another institution during the trial period for endometritis. We were also able to monitor the compliance to medication in the prophylaxis arm. On the other hand, the incidence of endometritis was much lower than previously estimated. It was unlikely that the 18 patients lost to follow up, equally distributed between both arms, would contribute to the overall incidence of endometritis. Consequently, a much larger sample size would be required for statistical power. However, the authors felt that there was already sufficient evidence for cessation of such prophylactic practices locally and prolonging the trial would not be the best use of existing resources. From a broader perspective, a low complication rate in women with ragged membranes overall, calls into question whether this finding should perturb the clinician.

The potential risk of bias due to a lack of placebo would have minimal impact on the primary outcome, since endometritis was defined by objective criteria such as the presence of fever, increased vaginal losses, leukocytosis and a positive genital culture.

\section{Conclusion}

Preventive use of antibiotics after vaginal delivery in women with ragged placental membranes did not result in a reduction of endometritis, ICU admission, transfusion or surgical evacuation. Advising women on the signs and symptoms of endometritis would suffice. In addition, the unnecessary use of antibiotics propagates medicalization of pregnancy, potentially alters fetal gut microbiome and should therefore be considered harmful.

\section{Additional file}

Additional file 1: Contains tabulated anonymized raw patient data in XLSX Worksheet format. (XLSX 64 kb)

\section{Abbreviations}

ICU: Intensive Care Unit; kg: kilograms; $\mathrm{m}^{2}$ : square metres; min:minute, NMRR: National Medical Research Register; WHO: World Health Organization

\section{Acknowledgements}

The authors would like to acknowledge the contributions of Dr. Norhashimah Ramlee and Dr. Mah Jia Mien for providing administrative and technical support in ensuring the timely completion of this paper. We also record our appreciation to the Director-General of Health Malaysia for permission for publication.

\section{Authors' contributions}

VHY conceived, drafted the manuscript and provided statistical analysis. PJY and TLN were involved in preparation of manuscript and acquisition of data. HNS was involved in the conception, design of the trial and supervision. TWS was involved in the conception, design and acquisition of data. All authors were responsible for revising the final manuscript. All authors read and approved the final manuscript.

Funding

Not applicable.

\section{Availability of data and materials}

The dataset supporting the conclusions of this article is included within the article and as additional files (Additional file 1).

\section{Ethics approval and consent to participate}

Ethical approval was granted by the Medical Research \& Ethics Committee of Malaysia (NMRR ID 16-1016-31034). Informed consent was obtained from all patients during recruitment.

\section{Consent for publication}

Obtained from all patients and Director-General of Health, Malaysia.

\section{Competing interests}

None of the authors have received any form of honoraria, funding, grant, employment or have financial interest in the manufacturer of Co-Amoxiclav ${ }^{\mathrm{TM}}$ (Amoxycillin + Clavulanic acid), Pharmaniaga (Shah Alam, Malaysia).

\section{Author details}

'Department of Obstetrics and Gynaecology, Sarawak General Hospital, Hospital Road, 93586 Kuching, Sarawak, Malaysia. ²Department of Obstetrics and Gynaecology, Sri Aman Hospital, Hospital Road, 95000 Sri Aman, Sarawak, Malaysia. ${ }^{3}$ Department of Obstetrics and Gynaecology, Bintulu Hospital, Nyabau Road, 97000 Bintulu, Sarawak, Malaysia. ${ }^{4}$ Department of Obstetrics and Gynaecology, Faculty of Medicine and Health Sciences, Universiti Malaysia Sarawak, 94300 Kota Samarahan, Sarawak, Malaysia.

Received: 8 May 2018 Accepted: 24 June 2019

Published online: 11 July 2019

References

1. Knight M, Kenyon S, Brocklehurst P, Neilson J, Shakespeare J, Kurinczuk JJ. On behalf of MBRRACE-UK. Saving lives, improving mothers' care - lessons learned to inform future maternity care from the UK and Ireland confidential enquiries into maternal deaths and morbidity 2009-12. Oxford: National Perinatal Epidemiology Unit, University of Oxford; 2014.

2. Division of Family Health Development. Report on the Confidential Enquiries into Maternal Deaths in Malaysia 2006-8. Ministry of Health Malaysia 2013

3. RCOG. Bacterial Sepsis Following Pregnancy. Green-top Guideline No.64b. Royal College of Obstetricians and Gynaecologists 2012. https://www.rcog org.uk/en/quidelines-research-services/quidelines/gtg64b ( Accessed 6/6) 2016). 
4. Meaney-Delman D, Bartlett LA, Gravett MG, Jamieson DJ. Oral and intramuscular treatment options for early postpartum endometritis in lowresource settings. A systematic review. Obstet Gynecol. 2015;125(4):789-800.

5. World Health Organization. Managing Puerperal Sepsis in: Midwifery education modules. 2nd edition. WHO Press, Geneva 2008. https://www. who.int/maternal_child_adolescent/documents/4_9241546662/en/. (Accessed 16/6/2016).

6. Newton ER, Prihoda TJ. Gibbs RS. A clinical and microbiologic analysis of risk factors for puerperal endometritis. Obstet Gynecol. 1990;75(3 Pt 1):402-6.

7. Varney H, Kriebs JM, Gegor CL. Varney'S midwifery $4^{\text {th }}$ edition. Jones \& Bartlett Publishers 2004: 1265-1266.

8. Coad J, Dunstall M, McCandlish R. Anatomy and physiology for midwives $2^{\text {nd }}$ edition: Elsevier; 2005. p. 193.

9. International Classification of Diseases, $10^{\text {th }}$ Edition: 2016 http://apps.who. int/classifications/icd10/browse/2016/en (Accessed 6/6/2016).

10. WHO recommendations for the prevention and treatment of postpartum haemorrhage 2012. http://apps.who.int/iris/bitstream/10665/75411/1/ 9789241548502_eng.pdf (Accessed 6/6/2016).

11. van Schalkwyk J, Van Eyk N. Antibiotic prophylaxis in obstetric procedures. J Obstet Gynaecol Can. 2010;32(9):878-92.

12. Chongsomchai C, Lumbiganon P, Laopaiboon M. Prophylactic antibiotics for manual removal of retained placenta in vaginal birth. Cochrane Database Syst Rev2014;(10):CD004904.doi: https://doi.org/10.1002/14651858.CD004904.

13. Chibueze EC, Parsons AJ, Ota E, Swa T, Oladapo OT, Mori R. Prophylactic antibiotics for manual removal of retained placenta during vaginal birth: a systematic review of observational studies and meta-analysis. BMC Pregnancy Childbirth. 2015;15:313. https://doi.org/10.1186/s12884-015-0752-4.

14. Liabsuetrakul T, Choobun T, Peeyananjarassri K, Islam QM. Antibiotic prophylaxis for operative vaginal delivery. Cochrane Database Syst Rev 2014; (10):CD004455. doi: https://doi.org/10.1002/14651858.CD004455.

15. Fernandez $H$, Gagnepain A, Bourget $P$, et al. Antibiotic prophylaxis against postpartum endometritis after vaginal delivery: a prospective randomized comparison between Amox-CA (Augmentin ${ }^{\mathrm{TM}}$ ) and abstention. Eur I Obstet Gynecol Reprod Biol. 1993;50(3):169-75.

16. Thinkhamrop J, Hofmeyr GJ, Adetoro O, Lumbiganon P, Ota E. Antibiotic prophylaxis during the second and third trimester to reduce adverse pregnancy outcomes and morbidity. Cochrane Database of Syst Rev. 2015; (6):CD002250. https://doi.org/10.1002/14651858.CD002250.

17. Mackeen AD, Packard RE, Ota E, Speer L. Antibiotic regimens for postpartum endometritis. Cochrane Database of Syst Rev. 2015;(2):CD001067. https://doi. org/10.1002/14651858.CD001067.

18. Incerpi MH. Chorio-Amnionitis and postpartum endometritis. In: Goodwin TM, Montoro MN, editors. Management of Common Problems in obstetrics and gynecology. West Sussex: Wiley-Blackwell; 2010. p. 50-2.

19. Dupont WD, Plummer WD. Power and sample size calculations: a review and computer program. Control Clin Trials. 1990;11(2):116-28.

20. Kenyon S, Pike K, Jones DR, et al. Childhood outcomes after prescription of antibiotics to pregnant women with spontaneous preterm labour: 7-year follow up of the ORACLE II trial. Lancet. 2008;372(9646):1319-27.

21. Azad MB, Konya T, Persaud RR. Impact of maternal intrapartum antibiotics, method of birth and breastfeeding on gut microbiota during the first year of life: a prospective cohort study. Br J Obstet Gynaecol. 2016;123(6):983-93.

\section{Publisher's Note}

Springer Nature remains neutral with regard to jurisdictional claims in published maps and institutional affiliations.

Ready to submit your research? Choose BMC and benefit from:
- fast, convenient online submission
- thorough peer review by experienced researchers in your field
- rapid publication on acceptance
- support for research data, including large and complex data types
- gold Open Access which fosters wider collaboration and increased citations
- maximum visibility for your research: over 100M website views per year
At BMC, research is always in progress.
Learn more biomedcentral.com/submissions

